\title{
HIF inhibitor topotecan has a neuroprotective effect in a murine retinal ischemia-reperfusion model
}

\author{
Hiromitsu Kunimi $^{1,2}$, Yukihiro Miwa ${ }^{1,2}$, Yusaku Katada ${ }^{1,2}$, Kazuo Tsubota ${ }^{\text {Corresp., }}$, Toshihide Kurihara ${ }^{\text {Corresp. } 1,2}$ \\ 1 Department of Ophthalmology, School of Medicine, Keio University, Tokyo, Japan \\ 2 Laboratory of Photobiology, School of Medicine, Keio University, Tokyo, Japan \\ Corresponding Authors: Kazuo Tsubota, Toshihide Kurihara \\ Email address: tsubota@z3.keio.jp, kurihara@z8.keio.jp
}

Purpose. The therapeutic approach for retinal ganglion cell (RGC) degeneration has not been fully established. Recently, it has been reported that hypoxia-inducible factor (HIF) may be involved with retinal neurodegeneration. In this study, we investigated neuroprotective effects of a HIF inhibitor against RGC degeneration induced in a murine model of retinal ischemia-reperfusion (I/R).

Methods. 8-weeks-old male C57/BL6J mice were treated with intraperitoneal injection of a HIF inhibitor topotecan $(1.25 \mathrm{mg} / \mathrm{kg})$ for 14 days followed by a retinal I/R procedure. 7 days after the I/R injury, the therapeutic effect was evaluated histologically and electrophysiologically.

Results. The increase of HIF-1a expression and the decrease of retinal thickness and RGC number in I/R were significantly suppressed by administration of topotecan. Impaired visual function in I/R was improved by topotecan evaluated with electroretinogram and visual evoked potentials.

Conclusions. Topotecan administration suppressed HIF-1a expression and improved RGC survival resulting in a functional protection against retinal I/R. These data indicated that the HIF inhibitor topotecan may have therapeutic potentials for RGC degeneration induced with retinal ischemia or high intraocular pressure. 


\section{HIF inhibitor topotecan has a neuroprotective effect in a 2 murine retinal ischemia-reperfusion model}

3

4 Hiromitsu Kunimi ${ }^{1,2}$, Yukihiro Miwa ${ }^{1,2}$, Yusaku Katada ${ }^{1,2}$, Kazuo Tsubota ${ }^{1}$ and Toshihide

5 Kurihara ${ }^{1,2}$

6

$7 \quad{ }^{1}$ Department of Ophthalmology, School of Medicine, Keio University, Tokyo, Japan

$8 \quad{ }^{2}$ Laboratory of Photobiology, School of Medicine, Keio University, Tokyo, Japan 9

10 Corresponding Authors:

11 Toshihide Kurihara ${ }^{1,2}$

12 Email address: kurihara@z8.keio.jp

13

14 Kazuo Tsubota ${ }^{1}$

15 Email address: tsubota@z3.keio.jp 


\section{Abstract}

17 Purpose. The therapeutic approach for retinal ganglion cell (RGC) degeneration has not been fully

18 established. Recently, it has been reported that hypoxia-inducible factor (HIF) may be involved

19 with retinal neurodegeneration. In this study, we investigated neuroprotective effects of a HIF

20 inhibitor against RGC degeneration induced in a murine model of retinal ischemia-reperfusion

$21(\mathrm{I} / \mathrm{R})$.

22 Methods. 8-weeks-old male C57/BL6J mice were treated with intraperitoneal injection of a HIF

23 inhibitor topotecan $(1.25 \mathrm{mg} / \mathrm{kg})$ for 14 days followed by a retinal $\mathrm{I} / \mathrm{R}$ procedure. 7 days after the

$24 \mathrm{I} / \mathrm{R}$ injury, the therapeutic effect was evaluated histologically and electrophysiologically.

25 Results. The increase of HIF-1 $\alpha$ expression and the decrease of retinal thickness and RGC number

26 in I/R were significantly suppressed by administration of topotecan. Impaired visual function in

27 I/R was improved by topotecan evaluated with electroretinogram and visual evoked potentials.

28 Conclusions. Topotecan administration suppressed HIF-1 $\alpha$ expression and improved RGC

29 survival resulting in a functional protection against retinal I/R. These data indicated that the HIF

30 inhibitor topotecan may have therapeutic potentials for RGC degeneration induced with retinal

31 ischemia or high intraocular pressure. 
32

33

34

35

36

37

38

\section{Introduction}

Tissue ischemia and hypoxia may induce irreversible neuronal degeneration. In the eye, retinal ganglion cell (RGC) degeneration is observed accompanied with ischemia in central retinal artery occlusion and ischemic optic neuropathy. Recently, optical coherence tomography (OCT) angiography technology reveals that capillary dropout is correlated with the decreased RGC layer thickness and visual field defects in glaucoma (Takusagawa et al., 2017). Mounting evidence suggests that retinal ischemia plays an important role in RGC degeneration (Wang et al., 2001). These damages directly affect visual acuity and visual field; however, therapeutic options for RGC degeneration are limited and establishment of a protective approach for RGC is desired.

42

Hypoxia-inducible factor (HIF) is a transcriptional factor that has a pivotal role in cellular adaptive response to hypoxic condition. Stabilization and activation of HIF induces cell survival under hypoxia including neovascularization, cell respiration, apoptosis, glucose metabolism, and embryogenesis (Semenza, 2011). In the retina, HIF plays a critical role in development, physiology and pathology related with angiogenesis and anaerobic metabolism (Kurihara et al., 2016; Kurihara, 2018). It has also reported that HIF-1 $\alpha$ expression is increased in human glaucomatous retina (Tezel \& Wax, 2004) suggesting a correlation between chronic RGC degeneration and HIF activation.

1 Retinal ischemia-reperfusion $(\mathrm{I} / \mathrm{R})$ is a well-established animal model to induce RGC degeneration 2 (Sellés-Navarro et al., 1996; Vidal-Sanz et al., 2001; Hartsock et al., 2016; Liu et al., 2019). This 3 model mimics an acute retinal artery occlusion such as CRAO or RGC death such as glaucoma. 54 A previous report showed a neuroprotective effect of carnosine against I/R with decrease of HIF- 
$551 \alpha$ expression in the retina ( $\mathrm{Ji}$ et al., 2014). However, the relation between retinal 56 neurodegeneration and HIF remains unclear. Topotecan is a topoisomerase inhibitor and is also 57 known as a potent HIF inhibitor (Rapisarda et al., 2002). Recently, we reported that topotecan 58 prevented retinal neovascularization and impaired visual function in a murine model of oxygen59 induced retinopathy (Miwa et al., 2019), while it remains unclear that pharmacological HIF 60 inhibition is effective for RGC degeneration. In this study, we investigated the protective effect of 61 topotecan for RGC in a murine model of retinal I/R. 


\section{Materials \& Methods}

\section{Ethics of animal research}

64 All procedures for animal experiments were approved by IACUC of Keio University (Approval

65 Number 2808), and were in accordance with NIH guidelines for work with animals, ARVO 66 statement for the Use of Animals in Ophthalmic and Vision Research and ARRIVE guidelines.

67 Abbreviations: IACUC, Institutional Animal Care and Use Committee; NIH, National Institutes 68 of Health; ARVO, Association for Research in Vision and Ophthalmology; ARRIVE, Animal 69 Research: Reporting of In Vivo Experiments.

\section{Drug administration}

72 All experiments were carried out with 8-weeks-old male C57/BL6J mice (CLEA Japan, Japan). 73 Animals were divided into two groups and intraperitoneally injected with phosphate buffered 74 saline (PBS) or Topotecan dissolved in PBS (1.25mg/kg, \#14129, Cayman Chemical, United 75 States) once per day for 14days prior to the retinal I/R. All mice were maintained on a standard 76 rodent diet (MF, Oriental Yeast Co., Ltd, Japan) and given free access to water. All cages were 77 maintained under controlled lighting (12h light/12h dark).

78

\section{Murine retinal I/R experiment}

80 The murine model of retinal $\mathrm{I} / \mathrm{R}$ followed with Western blotting, qPCR, retinal thickness 81 evaluation, RGC retrograde labeling and electrophysiological evaluation were performed as 82 previously described (Kunimi et al., 2019). Specifically, primary antibodies mouse anti-HIF-1 $\alpha$ 83 (1:1500; \#36169, CST, United States) and mouse anti- $\beta$-actin (1:4000; \#A5316, Sigma-Aldrich, 
84 United States) were used for Western blotting. The primers' sequences (Thermo Fisher Scientific, 85 United States) used for qPCR were also described in Kunimi et al., 2019.

86

\section{Statistical analysis}

88 The data were presented as the mean \pm SD. Comparison of two experimental conditions was

89 evaluated using Mann-Whitney’s U-test. A $p<0.05$ was considered statistically significant. 
90

91

92

93

94

95

96

97

98

99

100

101

102

103

104

105

106

107

108

109

110

111

112

\section{6}

\section{Results}

\section{Expression of HIF-1 $\alpha$ and its target genes after retinal I/R injury}

In the current study, we examined murine retinal I/R model to induce RGC degeneration. HIF-1 $\alpha$ protein expression in the retina was increased in 6hours after I/R injury (Fig 1A) with significant

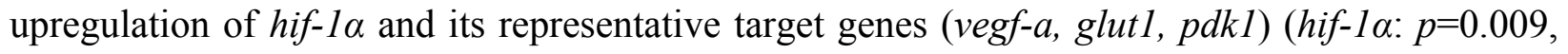
vegf-a: $p=0.009$, glut $1: p=0.009, p d k 1: p=0.009$, respectively) (Fig 1B). These data indicated that retinal HIF-1 $\alpha$ signaling was activated with I/R injury.

\section{Change of HIF-1 $\alpha$ and target gene expressions with topotecan administration}

Next, we administered topotecan intraperitoneally in order to inhibit HIF-1 $\alpha$ pharmacologically in mice. The increased HIF-1 $\alpha$ protein expression in post-I/R retinas $(p=0.009)$ was significantly $(p=0.009)$ suppressed in topotecan-treated mice compared to controls (Fig 2A, B). The upregulated retinal hif-1 $\alpha$ and the target genes were also significantly suppressed except for $p d k 1$ in treated mice compared to controls (hif-1 $\alpha: p=0.009$, vegf-a: $p=0.016$, glut1: $p=0.009, p d k 1: p=0.028$, respectively) (Fig 2C). These results suggested that systemic administration of topotecan inhibited increased HIF-1 $\alpha$ and upregulated target gene expression in post I/R retinas.

\section{Improvement of RGC survival with topotecan administration in post-I/R retinas}

We examined the retinal thickness to evaluate the effect of topotecan morphologically with OCT. Total retinal thickness was significantly $(p=0.021)$ thinner in a week after $\mathrm{I} / \mathrm{R}$ injury, while topotecan group showed significantly $(p=0.021)$ thicker retina compared to control (Fig 3). We further examined fluorogold retrograde labeling of RGCs to assess the cell survival 7 day after I/R injury. While the number of RGCs were significantly $(p=0.009)$ decreased in post-I/R retinas, 
113 topotecan administration significantly ( $p=0.009$ ) suppressed the decrease of RGC number (Fig 4).

114 These results indicated that topotecan administration had a neuroprotective effect improving RGC

115 survival against retinal $\mathrm{I} / \mathrm{R}$ damage.

116

117 Protective effect of topotecan for the impaired visual function with I/R injury.

118 To evaluate the change of retinal function with topotecan treatment, we examined ERG after I/R 119 injury. In this study, ERG waveforms in three different stimulating conditions were recorded 7days 120 after $\mathrm{I} / \mathrm{R}$ injury. The amplitudes was significantly decreased in each condition after I/R injury (rod 121 b-wave: $p=0.009$, mix a-wave: $p=0.009$, mix b-wave: $p=0.009$, cone $b$-wave: $p=0.009$, 122 respectively), while topotecan administration suppressed the decrease of amplitudes with $I / R$ 123 injury except for cone b-wave (rod b-wave: $p=0.028$, mix a-wave: $p=0.016$, mix $\mathrm{b}$-wave: $p=0.006$, 124 cone b-wave: $p=0.056$, respectively) (Fig 5). In addition to ERG, we also assessed VEP to evaluate 125 the protective effect of topotecan in $\mathrm{I} / \mathrm{R}$ injury. $\mathrm{I} / \mathrm{R}$ injured mice showed a significant $(p=0.009)$ 126 decrease of amplitudes and a significantly $(p=0.009)$ prolonged implicit time. On the other hands, 127 the decrease of VEP amplitudes was significantly $(p<0.016)$ suppressed with topotecan 128 administration (Fig 6). These results suggested that topotecan had a neuroprotective effect against $129 \mathrm{I} / \mathrm{R}$ damage functionally. 


\section{Discussion}

131 In this study, we focused on the role of HIF-1 $\alpha$ in the RGC degeneration. The contribution of HIF

132 to RGC death or nerve fiber degeneration has not well been documented previously. In the current

133 study, RGC loss was observed after I/R injury accompanied with retinal excessive expression of

134 HIF-1 $\alpha$ while HIF-1 $\alpha$ inhibition with topotecan protected RGC morphologically and functionally

135 in $\mathrm{I} / \mathrm{R}$ injured retinas. To our best knowledge, this is the first study to show a protective effect of

136 HIF inhibitor to RGC degeneration. In the current study, we injected topotecan before the I/R

137 procedure to obtain the maximum effect of the drug at the onset of the model. Administration of

138 the drug after $\mathrm{I} / \mathrm{R}$ is required to prove the therapeutic concept for diseases in future studies.

139

140 To date, several neuroprotective materials for RGC degeneration have been reported. Synthetic

141 steroid showed RGC protection via suppressing the microglial inflammation (Sun et al., 2019).

142 Rapamycin, an antibiotic agent, promoted autophagy in the retina and improved RGC survival

143 (Russo et al., 2018). Other antibacterial drug, minocycline, had a neuroprotective effect in retinal

144 I/R (Huang et al., 2018). Bevacizumab, a human monoclonal antibody to VEGF which is

145 downstream of HIF-1 $\alpha$, also reduced RGC apoptosis in a rat model of I/R (Kohen et al., 2018). In

146 addition, some of dietary factors also showed retinal neuroprotective effects. Resveratrol enhanced

147 the survival of RGCs against I/R via anti-inflammatory action (Luo et al., 2018). Xue-Fu-Zhu-Yu,

148 one of traditional Chinese medicine protected RGC from retinal ischemia damage (Tan et al.,

149 2017). Thus, these substrates showing RGC protective effects with various mechanisms are

150 expected for clinical application.

151

Peer] reviewing PDF | (2019:05:37674:2:1:NEW 6 Sep 2019) 
152 There are some studies conducted to investigate pathways of RGC apoptosis. Caspases had been

153 explored to play an important role in neuronal cell apoptosis of the inner retinal layer in the early

154 stage of I/R (Lam, Abler \& Tso, 1999), while RGC necrosis was induced via extracellular signal-

155 regulated kinase 1/2-receptor-interacting protein kinase 3 pathway (Gao, Andreeva \& Cooper,

156 2014). Ca-phospholipid binding protein annexin A1 is shown to increase IL-1 $\beta$ expression

157 promoting RGC death via p65 pathway (Zhao et al., 2017).

158

159 As previously reported, the HIF-1 $\alpha$ expression in each cell type is functionally related to the 160 phenotype of angiogenesis physiologically and pathologically. Critical roles of HIF-1 $\alpha$ in retinal 161 neuron including RGC (Nakamura-Ishizu et al., 2012), muller cells (Lin et al., 2011), astrocytes, 162 and microglia (Kurihara et al., 2011) have been shown by utilizing cell type specific conditional 163 gene knockout technology. Although the specific cell type contributing to the effect of the drug 164 administration has not been identified at this point, we will explore the contribution of HIF in each 165 cell type in future studies. It has been reported that the activation of NRF2/HO-1 pathway (a 166 downstream of HIF-1 $\alpha$ ) in RGCs inhibited RGC degeneration in a rat retinal I/R model (Varga et 167 al., 2013; He et al., 2014). In a rat chronic high IOP model, HIF-1 $\alpha$ expression was increased in 168 muller cells and astrocytes but not in microglia (Ergorul et al., 2010). These results indicated that 169 the in vivo phenotype might not be mediated by a single cell type but by the sum of these 170 compensates.

171 Previously, we described that hypoxic retinal pigment epithelium induced degeneration of 172 photoreceptor cells altering glucose and lipid metabolism through HIF expression (Kurihara et al., 173 2016). In contrast, it has been reported that the expression of HIF downstream genes such as 174 Erythropoietin (Grimm et al., 2002; Sullivan, Kodali \& Rex, 2011) or Vegf (Foxton et al., 2013) 
175 have a neuroprotective effect against neuronal damages in the retina. Thus, further studies are

176 needed to conclude the contribution of HIF in the degenerative retina. HIF may be related to the

177 neurodegeneration in a cell autonomous manner such as apoptosis (Greijer \& van der Wall, 2004);

178 besides, non-cell autonomous mechanisms including recruitment of cytotoxic inflammatory cells

179 or activation of supporting cells can be considered to induce RGC damages (Zera \& Zastre, 2017).

180 HIF inhibition may suppress these negative reactions against the RGC survival in the process of

181 the degeneration. Since HIF is a transcription factor, further study should be required to elucidate

182 the downstream mechanism of HIF in the RGC degeneration. Taken together, a HIF inhibitor

183 topotecan had a neuroprotective effect for RGCs in retinal ischemia following hyper intraocular

184 pressure. This study suggested that pharmacological HIF inhibition may be possible candidates for

185 RGC degeneration induced with retinal ischemia or high intraocular pressure. 


\section{Conclusions}

187 In the current study, we hypothesized that HIF was involved with RGC degeneration in acute 188 retinal I/R. This is the first research to indicate that topotecan had a protective effect against retinal 189 I/R histologically and electrophysiologically suppressing elevated HIF-1 $\alpha$ expression. Although 190 further study is needed to show the specificity of HIF in retinal neurodegeneration, topotecan is a 191 possible drug to protect RGC from retinal ischemia and high intraocular pressure.

192

\section{Acknowledgements}

194 We thank H. Torii; S. Ikeda; Y. Hagiwara; K. Mori; E. Yotsukura; X Jiang; M. Ibuki; C. Shoda; 195 N. Ozawa; A. Ishida; K. Takahashi and K. Kurosaki for critical discussions. This work was 196 supported by Grants-in-Aid for Scientific Research (KAKENHI, number 15K10881 and 197 18K09424) from the Ministry of Education, Culture, Sports, Science and Technology (MEXT) to 198 Toshihide Kurihara. 
199 References

200 Ergorul C, Ray A, Huang W, Wang DY, Ben Y, Cantuti-Castelvetri I, Grosskreutz CL. 2010.

201 Hypoxia inducible factor-1 $\alpha$ (HIF-1 $\alpha)$ and some HIF-1 target genes are elevated in 202 experimental glaucoma. Journal of molecular neuroscience : MN 42:183-191. DOI:

$203 \quad 10.1007 / \mathrm{s} 12031-010-9343-\mathrm{Z}$.

204 Foxton RH, Finkelstein A, Vijay S, Dahlmann-Noor A, Khaw PT, Morgan JE, Shima DT, Ng Y-

205 S. 2013. VEGF-A is necessary and sufficient for retinal neuroprotection in models of 206 experimental glaucoma. The American journal of pathology 182:1379-1390. DOI:

$207 \quad 10.1016 /$ j.ajpath.2012.12.032.

208 Gao S, Andreeva K, Cooper NGF. 2014. Ischemia-reperfusion injury of the retina is linked to 209 necroptosis via the ERK1/2-RIP3 pathway. Molecular vision 20:1374-87.

210 Greijer AE, van der Wall E. 2004. The role of hypoxia inducible factor 1 (HIF-1) in hypoxia 211 induced apoptosis. Journal of clinical pathology 57:1009-1014. DOI:

$212 \quad 10.1136 /$ jcp.2003.015032.

213 Grimm C, Wenzel A, Groszer M, Mayser H, Seeliger M, Samardzija M, Bauer C, Gassmann M, 214 Remé CE. 2002. HIF-1-induced erythropoietin in the hypoxic retina protects against light215 induced retinal degeneration. Nature Medicine 8:718-724. DOI: 10.1038/nm723.

216 Hartsock MJ, Cho H, Wu L, Chen W-J, Gong J, Duh EJ. 2016. A Mouse Model of Retinal 217 Ischemia-Reperfusion Injury Through Elevation of Intraocular Pressure. Journal of 218 Visualized Experiments:e54065-e54065. DOI: 10.3791/54065.

219 He M, Pan H, Chang RCC, So KF, Brecha NC, Pu M. 2014. Activation of the Nrf2/HO-1

220 antioxidant pathway contributes to the protective effects of lycium barbarum 
221

222

223

224

225

226

227

228

229

230

231

232

233

234

235

236

237

238

239

240

241

242

polysaccharides in the rodent retina after ischemia-reperfusion-induced damage. PLoS ONE 9:1-11. DOI: 10.1371/journal.pone.0084800.

Heiduschka P, Schnichels S, Fuhrmann N, Hofmeister S, Schraermeyer U, Wissinger B, Alavi M V. 2010. Electrophysiological and histologic assessment of retinal ganglion cell fate in a mouse model for OPA1- associated autosomal dominant optic atrophy. Investigative Ophthalmology and Visual Science 51:1424-1431. DOI: 10.1167/iovs.09-3606.

Huang R, Liang S, Fang L, Wu M, Cheng H, Mi X, Ding Y. 2018. Low-dose minocycline mediated neuroprotection on retinal ischemia-reperfusion injury of mice. :367-378.

Ji YS, Park JW, Heo H, Park JS, Park SW. 2014. The neuroprotective effect of carnosine ( $\beta$ Alanyl-1-Histidine) on retinal ganglion cell following ischemia-reperfusion injury. Current Eye Research 39:634-641. DOI: 10.3109/02713683.2013.855235.

Kohen MC, Tatlipinar S, Cumbul A, Uslu Ü. 2018. The effects of bevacizumab treatment in a rat model of retinal ischemia and perfusion injury. Molecular vision 24:239-250.

Kunimi H, Miwa Y, Inoue H, Tsubota K, Kurihara T. 2019. A Novel HIF Inhibitor Halofuginone Prevents Neurodegeneration in a Murine Model of Retinal Ischemia-Reperfusion. International Journal of Molecular Sciences 20:3171. DOI: 10.3390/ijms20133171.

Kurihara T. 2018. Roles of hypoxia response in retinal development and pathophysiology. Keio Journal of Medicine 67:1-9. DOI: 10.2302/kjm.2017-0002-IR.

Kurihara T, Westenskow PD, Gantner ML, Usui Y, Schultz A, Bravo S, Aguilar E, Wittgrove C, Friedlander MSH, Paris LP, Chew E, Siuzdak G, Friedlander M. 2016. Hypoxia-induced metabolic stress in retinal pigment epithelial cells is sufficient to induce photoreceptor degeneration. :1-22. DOI: 10.7554/eLife.14319. 
243 Kurihara T, Westenskow PD, Krohne TU, Aguilar E, Johnson RS, Friedlander M. 2011.

244 Astrocyte pvhl and HIF- $\alpha$ isoforms are required for embryonic-to-adult vascular transition 245 in the eye. Journal of Cell Biology 195:689-701. DOI: 10.1083/jcb.201107029.

246 Lam TT, Abler AS, Tso MOM. 1999. Apoptosis and caspases after ischemia-reperfusion injury 247 in rat retina. Investigative Ophthalmology and Visual Science 40:967-975.

248 Lin M, Chen Y, Jin J, Hu Y, Zhou KK, Zhu M, Le Y-Z, Ge J, Johnson RS, Ma J-X. 2011.

249 Ischaemia-induced retinal neovascularisation and diabetic retinopathy in mice with 250 conditional knockout of hypoxia-inducible factor-1 in retinal Müller cells. Diabetologia $251 \quad 54: 1554-1566$. DOI: 10.1007/s00125-011-2081-0.

252 Liu W, Xia F, Ha Y, Zhu S, Li Y, Folorunso O, Pashaei-Marandi A, Lin P-Y, Tilton RG, Pierce 253 AP, Liu H, Zhang W. 2019. Neuroprotective Effects of HSF1 in Retinal Ischemia254 Reperfusion Injury. Investigative Opthalmology \& Visual Science 60:965. DOI: $255 \quad 10.1167 /$ iovs.18-26216.

256 Luo H, Zhuang J, Hu P, Ye W, Chen S, Pang Y, Li N, Deng C, Zhang X. 2018. Resveratrol 257 delays retinal ganglion cell loss and attenuates gliosis-related inflammation from ischemia258 reperfusion injury. Investigative Ophthalmology and Visual Science 59:3879-3888. DOI: 10.1167/iovs. 18-23806.

Miwa Y, Hoshino Y, Shoda C, Jiang X, Tsubota K, Kurihara T. 2019. Pharmacological HIF 261 inhibition prevents retinal neovascularization with improved visual function in a murine oxygen-induced retinopathy model. Neurochemistry International 128:21-31. DOI: https://doi.org/10.1016/j.neuint.2019.03.008.

264 Nakamura-Ishizu A, Kurihara T, Okuno Y, Ozawa Y, Kishi K, Goda N, Tsubota K, Okano H, 265 Suda T, Kubota Y. 2012. The formation of an angiogenic astrocyte template is regulated by 
the neuroretina in a HIF-1-dependent manner. Developmental Biology 363:106-114. DOI: 10.1016/j.ydbio.2011.12.027.

268

Rapisarda A, Uranchimeg B, Scudiero DA, Selby M, Sausville EA, Shoemaker RH, Melillo G. 2002. Identification of small molecule inhibitors of hypoxia-inducible factor 1 transcriptional activation pathway. Cancer Research 62:4316-4324.

Russo R, Varano GP, Adornetto A, Nazio F, Tettamanti G, Girardello R, Cianfanelli V, Cavaliere F, Morrone LA, Corasaniti MT, Cecconi F, Bagetta G, Nucci C. 2018. Rapamycin and fasting sustain autophagy response activated by ischemia/reperfusion injury and promote retinal ganglion cell survival. Cell Death and Disease 9. DOI: 10.1038/s41419-018-1044-5.

Sellés-Navarro I, Villegas-Perez MP, Salvador-Silva M, Ruiz-Gómez JM, Vidal-Sanz M. 1996. Retinal ganglion cell death after different transient periods of pressure-induced ischemia and survival intervals: A quantitative in vivo study. Investigative Ophthalmology and Visual Science 37:2002-2014.

Semenza GL. 2011. Regulation of Metabolism by Hypoxia-Inducible Factor 1. Cold Spring Harbor Symposia on Quantitative Biology 76:347-353. DOI: 10.1101/sqb.2011.76.010678.

Sullivan T, Kodali K, Rex TS. 2011. Systemic Gene Delivery Protects the Photoreceptors in the Retinal Degeneration Slow Mouse. Neurochemical Research 36:613-618. DOI: 10.1007/s11064-010-0272-6.

Sun H-J-Q, Xue D-D, Lu B-Z, Li Y, Sheng L-X, Zhu Z, Zhou Y-W, Zhang J-X, Lin G-J, Lin SZ, Yan G-M, Chen Y-P, Yin W. 2019. A Novel Synthetic Steroid of 2 $\beta, 3 \alpha, 5 \alpha$-Trihydroxyandrost-6-one Alleviates the Loss of Rat Retinal Ganglion Cells Caused by Acute 
288 289 290 291 292 293 294 295 296 297 298 299 300 301 302 303 304 305 306 307 308 309

Intraocular Hypertension via Inhibiting the Inflammatory Activation of Microglia. Molecules 24:252. DOI: 10.3390/molecules24020252.

Takusagawa HL, Liu L, Ma KN, Jia Y, Gao SS, Zhang M, Edmunds B, Parikh M, Tehrani S, Morrison JC, Huang D. 2017. Projection-Resolved Optical Coherence Tomography Angiography of Macular Retinal Circulation in Glaucoma. Ophthalmology 124:1589-1599. DOI: 10.1016/j.ophtha.2017.06.002.

Tan SQ, Geng X, Liu JH, Pan WHT, Wang LX, Liu HK, Hu L, Chao HM. 2017. Xue-fu-Zhu-Yu decoction protects rats against retinal ischemia by downregulation of HIF-1 $\alpha$ and VEGF via inhibition of RBP2 and PKM2. BMC Complementary and Alternative Medicine 17:1-16. DOI: $10.1186 / \mathrm{s} 12906-017-1857-2$.

Tezel G, Wax MB. 2004. Hypoxia-inducible factor $1 \alpha$ in the glaucomatous retina and optic nerve head. Archives of Ophthalmology 122:1348-1356. DOI: 10.1001/archopht.122.9.1348.

Varga B, Gesztelyi R, Bombicz M, Haines D, Szabo AM, Kemeny-Beke A, Antal M, Vecsernyes M, Juhasz B, Tosaki A. 2013. Protective effect of alpha-melanocyte-stimulating hormone $(\alpha-\mathrm{MSH})$ on the recovery of ischemia/reperfusion (I/R)-induced retinal damage in a rat model. Journal of Molecular Neuroscience 50:558-570. DOI: 10.1007/s12031-0139998-3.

Vidal-Sanz M, Lafuente MP, Mayor S, De Imperial JM, Villegas-Pérez MP. 2001. Retinal ganglion cell death induced by retinal ischemia: Neuroprotective effects of two alpha-2 agonists. Survey of Ophthalmology 45:261-267. DOI: 10.1016/S0039-6257(01)00205-3.

Wang L, Cioffi GA, Cull G, Dong J, Fortune B. 2001. Immunohistologic Evidence for Retinal Glial Cell Changes in Human Glaucoma. :1088-1094. 
310 Zera K, Zastre J. 2017. Thiamine deficiency activates hypoxia inducible factor-1 $\alpha$ to facilitate 311 pro-apoptotic responses in mouse primary astrocytes. PloS one 12:e0186707-e0186707. 312 DOI: 10.1371/journal.pone.0186707.

313 Zhao Y, Li X, Gong J, Li L, Chen L, Zheng L, Chen Z, Shi J, Zhang H. 2017. Annexin A1 314 nuclear translocation induces retinal ganglion cell apoptosis after ischemia-reperfusion 315 injury through the p65/IL-1 $\beta$ pathway. Biochimica et Biophysica Acta - Molecular Basis of 316 Disease 1863:1350-1358. DOI: 10.1016/j.bbadis.2017.04.001. 


\section{Figure 1}

HIF-1 $\alpha$ and its target genesexpression in post- I/R retina.

(A) Western blots show retinal HIF-1 $\alpha$ expression is increased and maintained 6 hours after

I/R injury. (B) Hif-1 $\alpha$ and its representative target genes were upregulated in post-I/R retina detected by qPCR $(n=5)$. Gapdh was used as the internal control. Error bars indicate the standard error. Cont; control. ${ }^{* *} p<0.01$, Mann-Whitney's U test . 


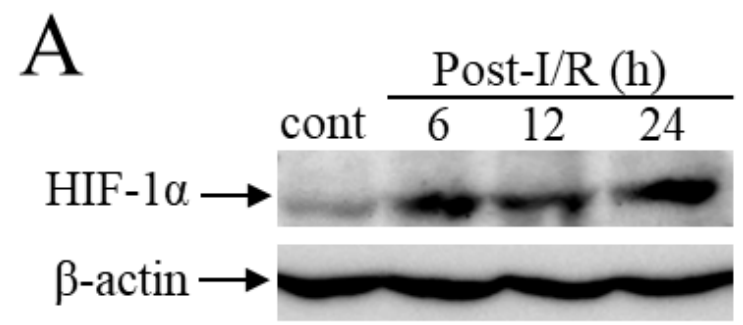

B

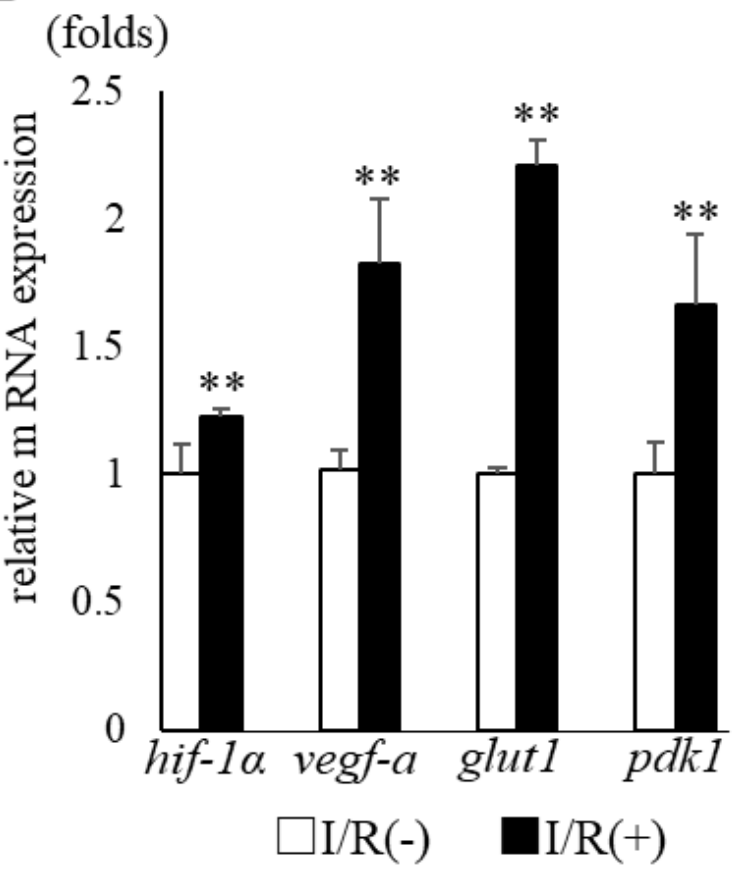




\section{Figure 2}

Topotecan administration suppresses increased HIF-1 $\alpha$ and upregulated targetgenes in $\mathrm{I} / \mathrm{R}$ retinas.

(A) Western blots for HIF-1 $\alpha$ and $\beta$-actin in control or I/R retinas with or without topotecan administration ( $n=5)$. (B) Quantification of the blots indicating that topotecan administration suppressed increased HIF-1 $\alpha$ expression. (C) Hif-1 $\alpha$ and its representative target genes detected by qPCR $(n=5)$. Note that upregulated genes were suppressed by topotecan administration. Gapdh was used as the internal control. Error bars indicate the standard error. ${ }^{*} p<0.05,{ }^{* *} p<0.01$, Mann-Whitney's U test. 
A

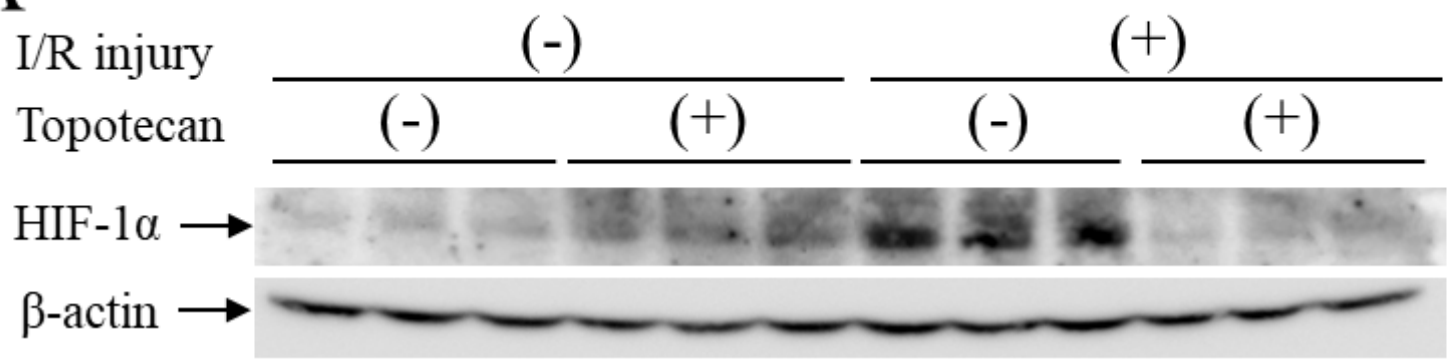

B

$\mathrm{C}$ (folds) hif-1a vegf-a glut1 pdk1
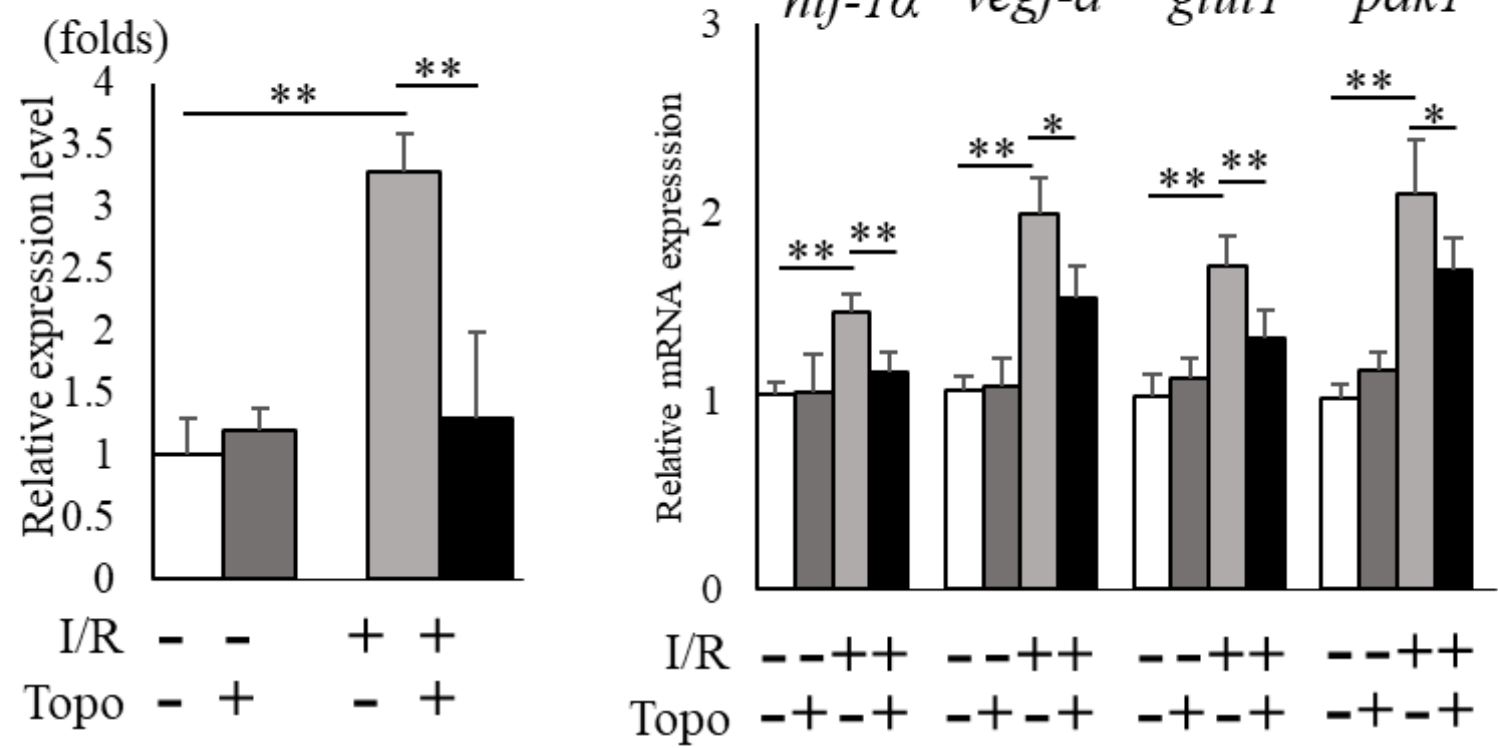
Figure 3

Evaluation of totalretinal thickness with OCT.

(A-D) Representative OCT images from each group. Scale bar; $100 \mu \mathrm{m}$. (E) The average of total retinal thickness quantified in OCT $(n=4)$. Note that decrease of retinal thickness was suppressed by topotecan administration post-I/R injury. Error bars indicate the standard error. ${ }^{*} p<0.05$, Mann-Whitney's $\mathrm{U}$ test. 

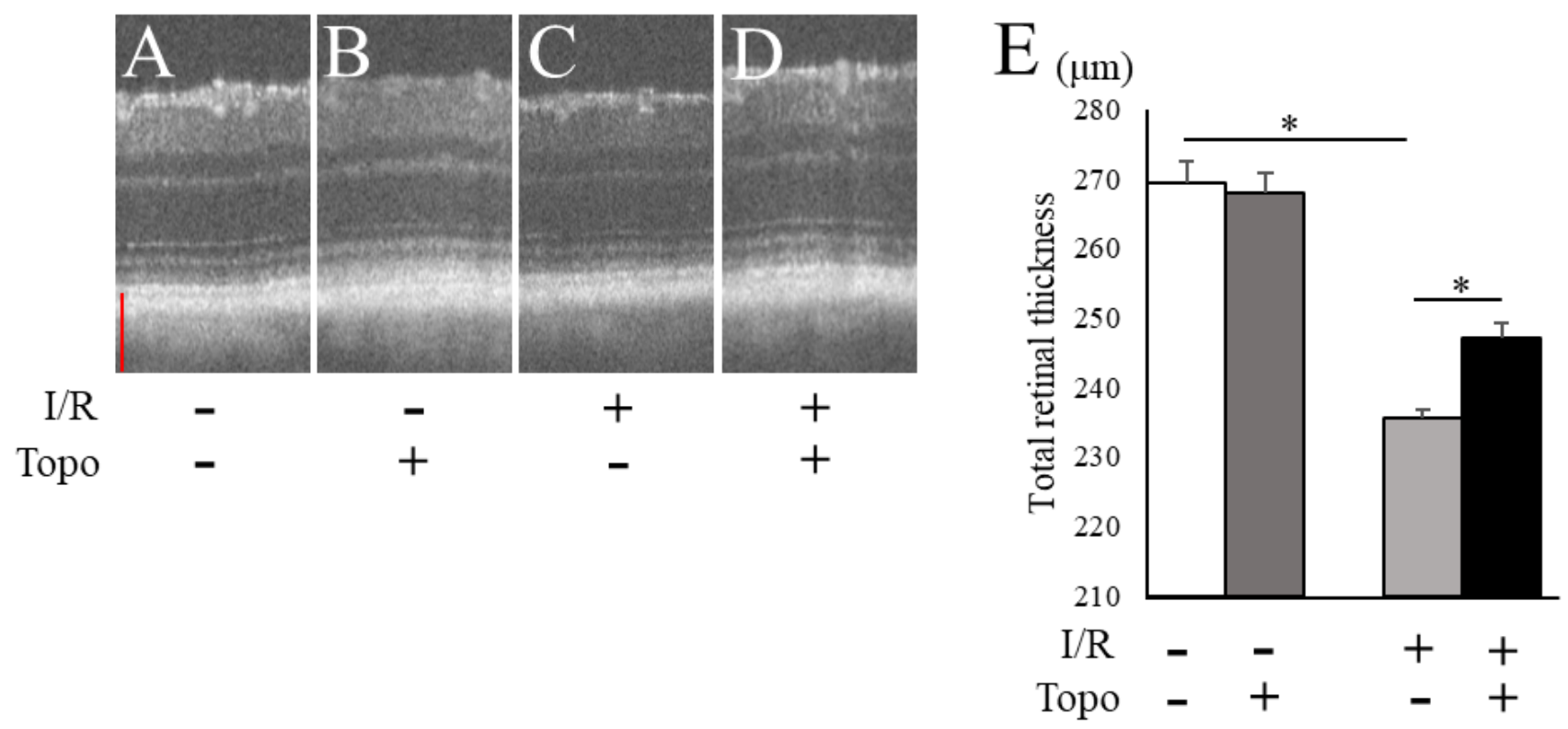


\section{Figure 4}

Fluorogold retrograde labeling of RGCs.

(A) A representative quadrant retina with fluorogold-labeled RGCs. $200 \mu \mathrm{m}$ square with red at $1 \mathrm{~mm}$ from optic disc head indicates the area for RGC densitometry. (B-E) Magnified images for control and post-I/R retina with or without topotecan administration . Scale bars; $200 \mu \mathrm{m}$ in quadrant retina, $50 \mu \mathrm{m}$ in magnified images. (F) The quantification of RGC density for each group $(n=5)$. Note that decrease of RGCs was suppressed by topotecan administration. Error bars indicate the standard error. ${ }^{* *} p<0.01$, Mann-Whitney's $U$ test . 

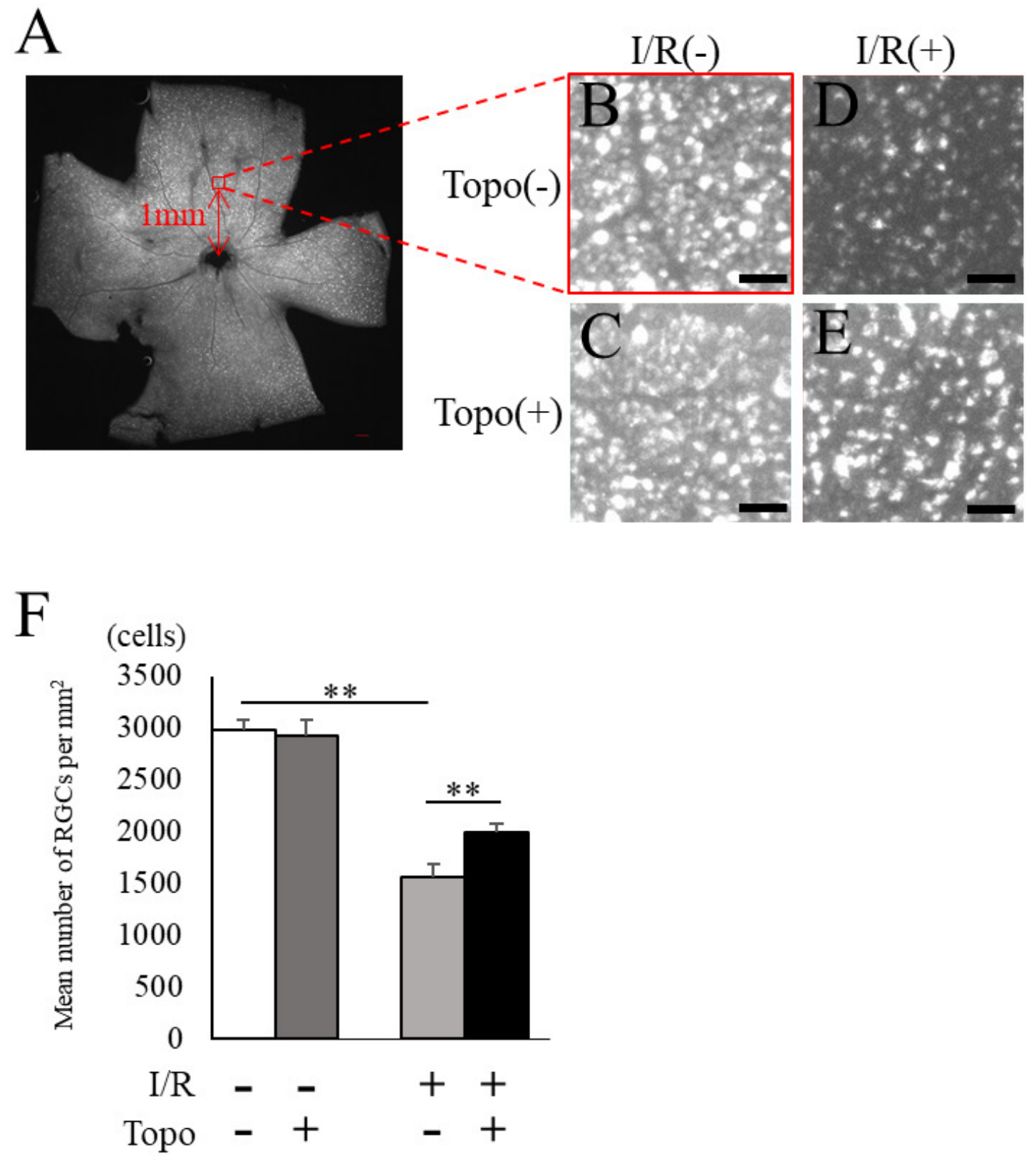


\section{Figure 5}

\section{Retinal functionevaluated with ERG.}

(A-C) Representative ERG waveforms for rod, mixed, and cone conditions. Black arrows indicate the timing of the stimulation. The averaged amplitudes were shown for rod b-wave (D), mixed a-wave (E), mixed bwave $(F)$, and cone b-wave (G) $(n=5-6)$. Note that most of decreased amplitudes were suppressed by topotecan administration. Error bars indicate the standard error. ${ }^{*} p<0.05,{ }^{* *} p<0.01$, Mann-Whitney's $U$ test 


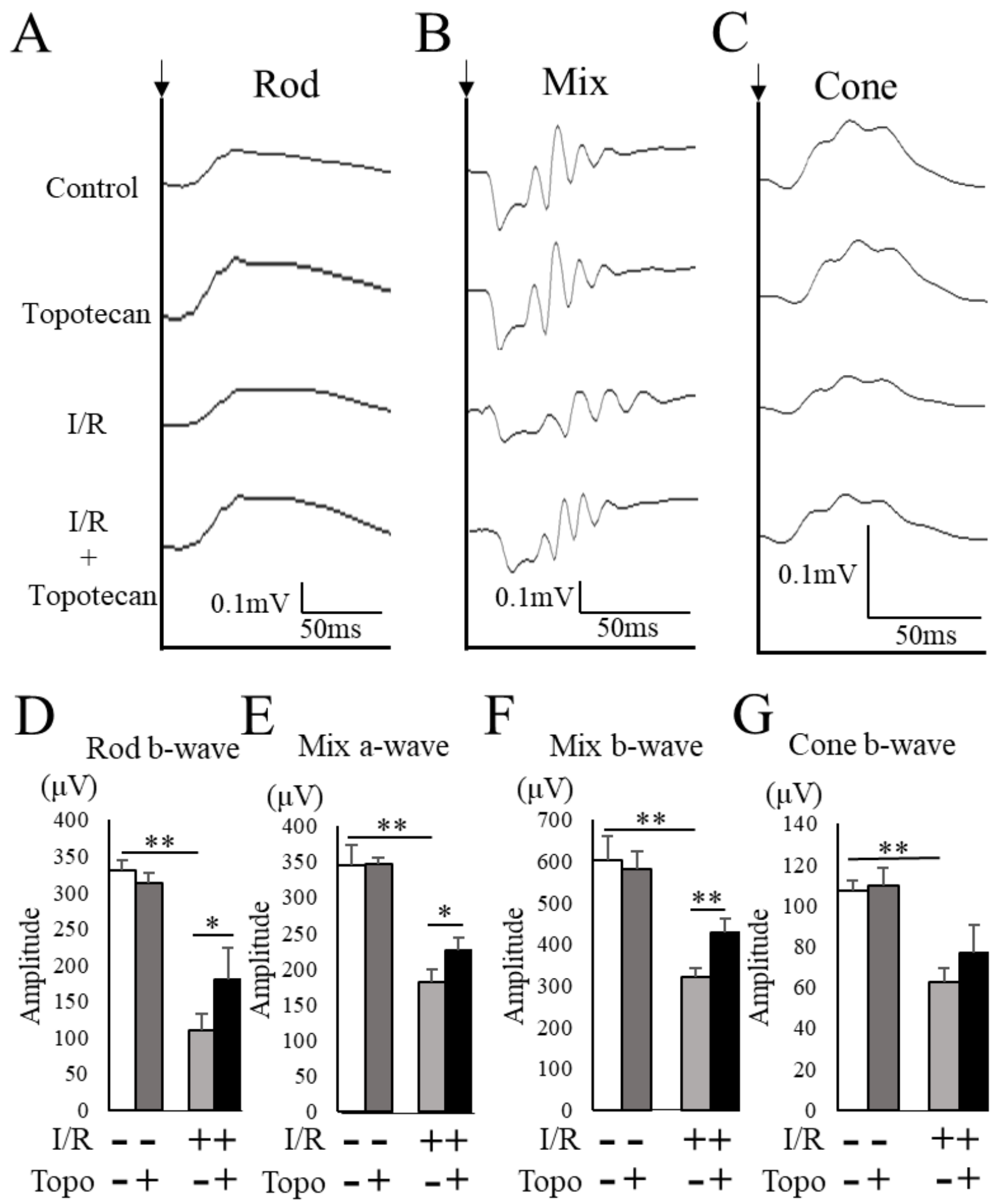




\section{Figure 6}

Evaluation of visual function detected with VEP.

(A) Representative VEP waveforms from control and post-I/R retina with or without topotecan administration . A black arrow indicates the timing of the stimulation. (B) The average of VEP amplitudes $(n=5)$. Note that decrease of VEP amplitude was suppressed by topotecan administration. (C) The average of VEP implicit time. Error bars indicate the standard error. ${ }^{*} p<0.05,{ }^{* *} p<0.01$, Mann-Whitney's U test . 


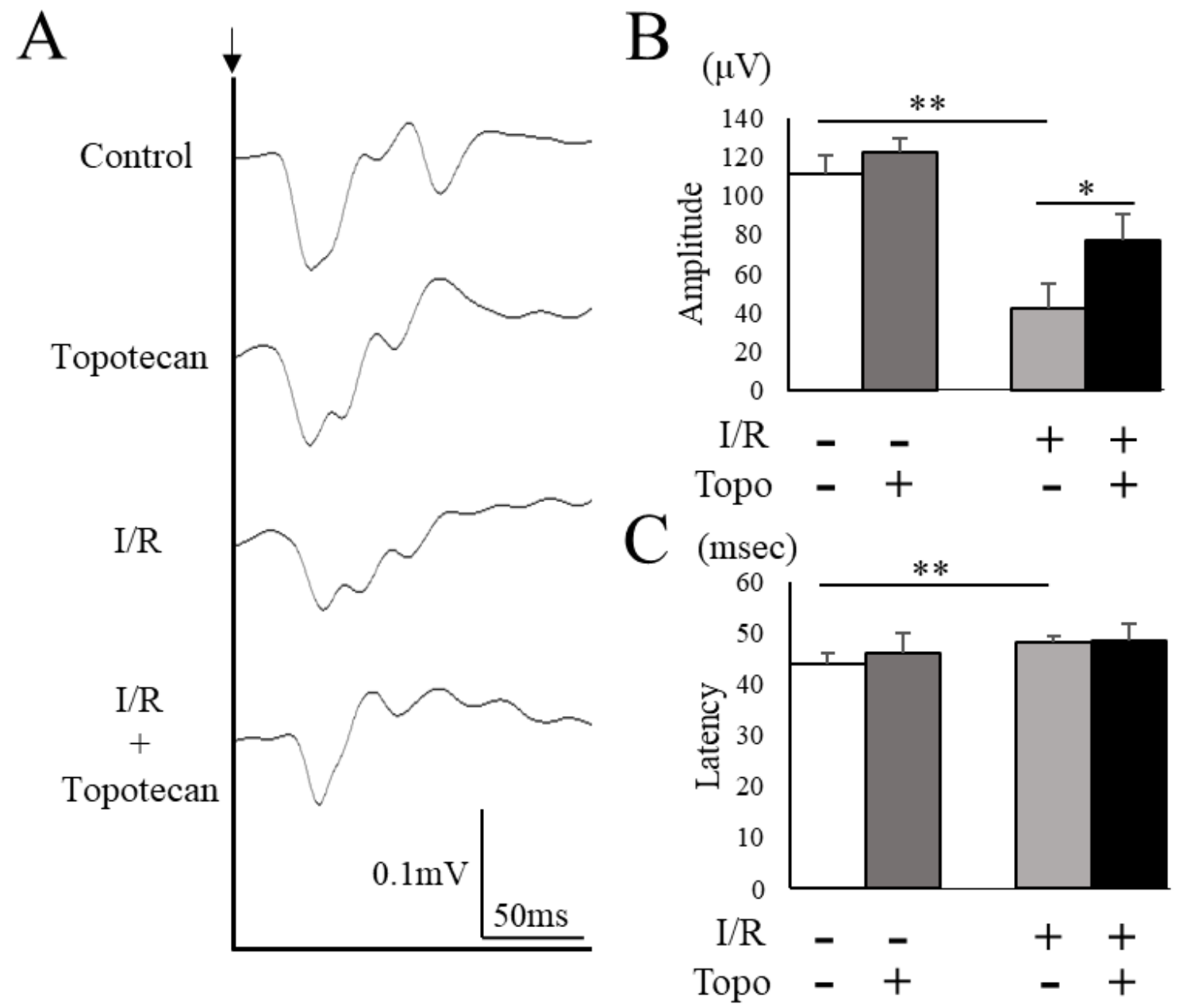

